\title{
On the strategy of ecological design from the perspective of ecological civilization construction
}

\author{
Fang $\mathrm{Gu}^{1 *}$, Chul-soo $\mathrm{Kim}^{2}$ \\ ${ }^{1}$ Department of Design, Hezou University, City, Province, 542899, China \\ ${ }^{1}$ Department of Industrial Design, Pukyong National University, Busan City, Province, 48513, Korea \\ ${ }^{2}$ Department of Industrial Design, Pukyong National University, Busan City, Province, 48513, Korea
}

\begin{abstract}
This paper takes the ecological design strategy of the construction of ecological civilization as the research topic, analyzes the connotation of ecological civilization and ecological design, and combines the relationship between ecological civilization and ecological design, adopts the methods of practical research, questionnaire survey, literature and other methods. This paper discusses that ecological design is the need and embodiment of ecological civilization construction, and then puts forward the strategy of developing ecological design on this basis. This paper proposes that designers should shoulder more social responsibility and practice ecological design, so as to introduce ecological awareness into public awareness.
\end{abstract}

\section{Introduction}

At present, due to the rapid development of economy, it leads to excessive consumption of natural resources, which gradually highlights the problem of human living environment. People began to pay attention to the environment and ecological situation and actively take various countermeasures, but the reality is not optimistic. The cruel reality of haze, garbage accumulation, water pollution, land deterioration and so on makes people start to reflect. Ecological civilization is not only a countermeasure put forward by human beings after reflecting on reality, but also a high-level pursuit of human existence. Nowadays, advanced countries, from natural science to Philosophy and social science, pay close attention to and study the strategies of ecological civilization construction. As one of the favorable measures for the construction of ecological civilization, ecological design is in the ascendant. It tries to start from the source of design, deal with the nature and performance of ecological problems, and act actively in theory and practice, so as to fundamentally solve the root cause of ecological crisis.

\section{The connotation of ecological civilization and ecological design}

\footnotetext{
${ }^{1}$ Associate professor, Hezhou University. Ph. D. Candidates, Department of Industrial Design, Pukyong National University

2 Professor, Department of Industrial Design, Pukyong National University
}

* Corresponding author's e-mail: 505665578@qq.com
Since mankind entered a civilized society, it has continuously transformed the physical and spiritual world, and has achieved rapid results. Since entering industrial civilization, the desire of humans to dominate and conquer nature has become stronger. They have even resorted to blind pursuit of immediate interests. Although the economy has developed rapidly in a relatively short period of time, it has led to a series of pollution and waste problems. And it is getting worse and worsening day by day, and then threatens the closely related living environment of human beings, which has caused a serious ecological crisis. Therefore, the relationship between humans and nature has become subtle, and humans have gradually come to understand that only by respecting nature, maintaining ecological balance, and actively understanding and accepting ecological civilization, can the path of harmony between man and nature be achieved. Ecological civilization, as a more advanced and advanced form of civilization than industrial civilization, is an extension and extension of social civilization in the natural environment. It is based on respecting and maintaining the value and order of the ecological environment, and focuses on the continued development of humanity in the future. , Emphasizes that starting from the maintenance of the overall interests of society, the economy, and the ecosystem, attaches importance to the limited support of resources and ecology, attaches importance to the mutual coordination and common development of human society and the natural world, and promotes the mainstream value 
orientation of human beings and social practices and natural ecological effects (Positive and negative effects) A view of civilized society that is consciously incorporated into all social and economic activities. Its essential requirement is to achieve the dual harmonious goal of man and nature and man and man, and then realize the sustainable development of society, economy and nature. 3

Art design is not only closely related to people's clothing, food, housing and transportation, but also can be said without exaggeration that art design is fully reflected in all aspects of life. Art and design affect the process of human civilization. From the early days of human life, people have the pursuit and design of beauty, one of the achievements of social civilization. In particular, industrial design encourages people to pursue newer, better and more advanced design and the wealth and energy per capita, which represent modernization. Driven by this kind of values, the designed products often have a very short service life and do not put the service life of the products in the first place. ${ }^{4}$ It goes against the principle that the ultimate goal of design is to design for the harmonious existence of human and nature.

In this case, mankind must break through the traditional thinking formula and carry out ecological design that is beneficial to the sustainable development of future generations. "According to the principle of the existence of the natural environment and interacting with and coordinating with nature, it has the least impact on the environment. A sustainable development design that can bear all signs of life is called ecological design." 5 The development trend of ecological design is to achieve ecological civilization as the goal, establish a sustainable development design concept, and maintain the green environment of the human earth for a long time. Therefore, it is also called green design or life cycle design or environmental design. Ecological design has become the mainstream of 21 st century design artists' pursuit of development.

\section{Ecological civilization and ecological design}

\subsection{Ecological design comes from the need of social civilization construction}

Ecological design can undoubtedly improve ecological problems from the source. First, ecological design can achieve the purpose of protecting the environment and curbing excessive consumption of natural resources. It is a long-term strategy for maintaining sustainable social and economic growth. Secondly, in industrial production, from materials to energy to commodity circulation, it can reduce the production cost of the enterprise as a whole, form a virtuous circle, avoid potential liability risks of the

\footnotetext{
3 Xiu Zhang. Philosophical thinking on the construction of ecological civilization [J]. Lanzhou journal, 2009 (2): 11-13

${ }^{4}$ Leitao Zhang. Ecological design and ecological products [J]. Gansu science and technology, 2005 (2): 179-180

5 Shoubing Wang, Wu Feng, Liu Jingru. Industrial Ecology

[M]. Beijing: Chemical Industry Press, 2006 (1): 80
}

enterprise, and ultimately improve the industry's own competitiveness. At present, many developing countries in the world are facing an ecological crisis in flight, the main reason is that humans 'excessive extraction of natural resources has led to the continuous destruction of the natural ecological environment. People always think about the rapid development of their own interests regardless of the ecological environment Affordability. For more than 30 years since the reform and opening up, China's development is in the middle stage of industrialization. With extensive industrial production methods, it has unswervingly sought to maximize profits. Premier Wen Jiabao of the State Council stated in the government work paper that "excessive consumption of energy resources and intensified environmental pollution" and "excessive cost of resources and environment for economic growth" are prominent contradictions and problems in the current economic and social development, which is fully in line with reality. ${ }^{6}$ Consumed a lot of resources for economic development, and ultimately restricted and affected the improvement of people's living standards. Therefore, the report of the 17th National Congress of the Communist Party of China clearly requires: "Build an ecological civilization, and basically form an industrial structure, growth mode, and consumption pattern that saves energy resources and protects the ecological environment. A circular economy has formed a larger scale, and the proportion of renewable energy has increased significantly. Pollutant emissions are effectively controlled, and the quality of the ecological environment is significantly improved. "The Chinese government emphasizes the importance of ecological design, comprehensively considers the factors of people, the environment, and resources, pays attention to the design of healthy development, and pays attention to sustainable development. In a resource-saving society, ecological design and ecological civilization construction are imminent.

\subsection{Sustainable design}

The key point of ecological civilization construction is to gradually transform the long-term conservative concept, and then to improve technology. As a comprehensive concept, the construction of ecological civilization must be realized by a series of specific measures, and ecological design must follow this strategic principle to carry out sustainable design. Ecological design plays an important role in the construction of ecological civilization. With the development of the times, people not only want to pursue higher quality of life on the basis of food, clothing and warmth, but also begin to pursue a safer, healthy and comfortable ecological design environment, so as to make rational use of natural resources, so as to improve the living environment of human beings in order, so that

\footnotetext{
6 Zhouqi Chen, Vice Chairman. Opening Speech at the Fifth Working Conference of the National Small and Medium-sized City Ecological Environment Construction Experimental Zone
} 
people really enjoy the fruits of ecological civilization. Ecological design requires designers to design from the perspective of sustainable development. When environmental factors are included in the design, they should try their best to reduce the adverse impact on the environment. The principle of sustainable development should be considered in all aspects of the design. Green environmental protection materials should be preferred in the design, and the discharge of harmful gases and sewage and the recovery of waste should be properly handled in the production process. In a word, the purpose of design is not only to meet people's daily needs, but also to improve people's overall quality of life and create a safe, healthy and comfortable living environment for people. It can be seen that ecological design is the specific practice and realization way of ecological civilization construction concept in the design field.

\section{The strategy of developing ecological design}

\subsection{Ecological design should pay attention to the selection of materials with ecological performance}

Materials are very important elements in the ecological design. Raw materials with renewable, low energy consumption and little impact on the environment are selected for design, especially those with ecological performance. On the one hand, recyclable materials that can be reused can be used to reduce the hazardous wastes generated in the process of production and use. On the other hand, green materials can be self degraded through the metabolism of the earth, and can improve the environmental ecological sustainability of product design. The principle of raw material selection in ecological design is: "whether to reduce the use of materials through design; avoid the use of materials under management or restriction, avoid the use of rare materials; reduce the type of materials, use the same or compatible materials for additional parts, reduce the incompatible materials; use recyclable and renewable materials, generally use single component materials as far as possible, avoid No composite materials, etc. "7 In the product design, we should try our best to use a large number of plant materials that grow naturally and are harmless to human body. In addition, the use of raw materials should conform to the applicability principle of ecological design.

\subsection{Ecological design should pay attention to saving energy of the earth}

Ecological design pays attention to the needs of various product functions, but also focuses on saving the earth's energy, reducing the waste of energy, repeatedly demonstrating and optimizing the design scheme and product performance, in the outer packaging design, it should consider the convenience of transportation and storage, improve the quality and use efficiency of products, improve the recovery rate and reuse rate of waste and other means to achieve. In particular, it is necessary to improve the quality and efficiency of the products so that they are easy to use and durable, and easy to repair and maintain. By optimizing the design, the standard modular structure is used to the maximum extent, so that the service life of the product can be extended through the immediate replacement of parts, so that when the service life of the product is over, the useful parts can be disassembled for continued use, so that the parts can be recycled, or converted to other uses. That is to say, ecological design not only requires that the designed products have the characteristics of high quality and high performance, but also that the raw materials, manufacturing and recycling should be ecological during the production and processing of products.

\subsection{Ecological design should pay attention to reduce environmental pollution}

Industrial production has a significant negative impact on the environment. For example, if the gas released by enterprises in processing and production is not purified and discharged directly, it will affect the air quality. The production process with large pollution and excessive consumption is also easy to cause adverse effects on the cleaning of water sources. If the waste generated by enterprises is not treated, it will change the composition of the soil in depth and cause soil Land pollution, as well as enterprises in the production and processing will cause heat to bring a series of greenhouse effects and so on. This requires designers to fully consider all aspects of environmental factors on the premise of meeting the design function and use requirements. Less resources are used in manufacturing, application and recycling, so the impact on the environment will be less, so as to avoid too much burden on the environment. Even in the early stage of design and development, designers should consider the negative impact of waste water, waste gas and waste in subsequent production, as well as the series of noise pollution, harmful trace element radiation and electromagnetic interference that will be brought. For example, in the packaging design, not only to reduce the consumption of packaging materials and energy, but also to consider that the used packaging materials are easy to identify in appearance, conducive to classification and recycling, renewable recycling after collection, and reduce the non degradable harmful substances.

\footnotetext{
${ }^{7}$ Zhongfan Zhou. Product life cycle design guide [M]. Beijing: China Environmental Science Press, 2006.01:146
} 


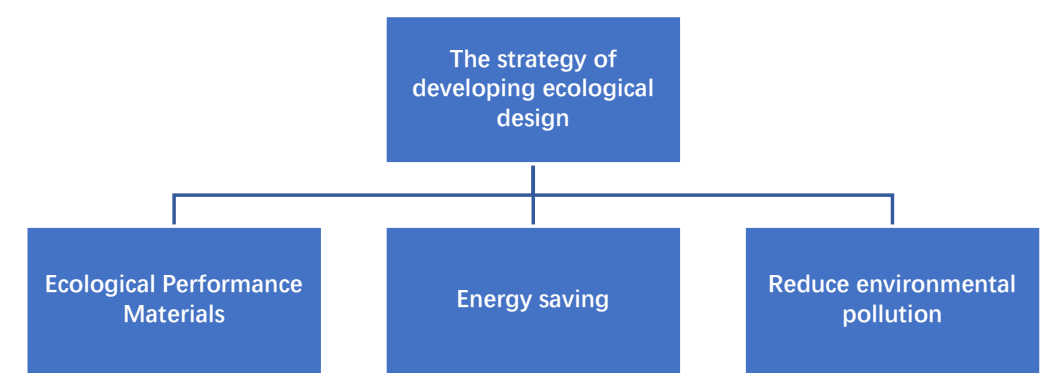

Figure 1. The strategy of developing ecological design

\section{Conclusion}

The construction of ecological civilization depends on the awakening of people's ecological consciousness and the active participation of the public. Ecological design pays attention to the sustainable development of resources and environment. Starting from the relationship of "people, things and environment", as a designer, he should shoulder social responsibility, and in the design behavior and design works, he should practice ecological design, so as to introduce ecological awareness into public awareness, change people's consumption concept, make ecological civilization a normal state, slow down the plunder of nature and reduce pollution Ranran, for the development of future generations to leave space for natural resources and a better living environment.

\section{Reference:}

1. Xiu Zhang. Philosophical thinking on the construction of ecological civilization [J]. Lanzhou journal, 2009 (2): 11-13

2. Leitao Zhang. Ecological design and ecological products [J]. Gansu science and technology, 2005 (2): $179-180$

3. Shoubing Wang, Wu Feng, Liu Jingru. Industrial Ecology [M]. Beijing: Chemical Industry Press, 2006 (1): 80

4. Zhouqi Chen, Vice Chairman. Opening Speech at the Fifth Working Conference of the National Small and Medium-sized City Ecological Environment Construction Experimental Zone

5. Zhongfan Zhou. Product life cycle design guide [M]. Beijing: China Environmental Science Press, 2006.01:146

6. junhang Wang 's construction strategy of Guangzhou ecological water city from the perspective of water ecological civilization construction [J]. Housing and real estate, 2019(7): 215

7. Yao Cheng: design practice of handicraft industry from the perspective of ecological civilization construction [J]. Journal of Changchun Normal University, 2017 (4): 192-194 